\title{
Psychopathology
}

Bormuth, M. 281

Ferri, F. 330

Fuchs, T. 279, 301

Gallese, V. 330

Henriksen, M.G. 320

Herpertz, S. 279

Luft, S. 345

Mishara, A.L. 309
Parnas, J. 320

Rosfort, R. 337

Schlimme, J.E. 345, 355

Schwartz, M.A. 289, 309

Stanghellini, G. 337

Vogeley, K. 295

Wiggins, O.P. 289

\section{Subject Index Vol. 46, No. 5, 2013}

Ambiguity 295

Analogical appresentation 345

Applied phenomenology 345

Autonomy 337

Berze 309

Binswanger 309

Communication 345

Compliance 320

Conrad 309

Consciousness 309

Coping 355

Core disturbance (Grundstörung) 309

Delusions 309

Doctor-patient-relationship 355

Eidetic phenomenology 345

Empathy 337, 345

Ethics 337

Evidence 289

Existence 301

Existential counseling 355

- philosophy 355

'Explaining' ('Erklären') 295
Freedom 281

Friedrich Nietzsche 281

Gehäuse 355

'General Psychopathology' ('Allgemeine Psychopathologie') 295

Grenzsituation 355

Gruhle 309

Heidelberg School of Psychiatry 309

Helen Longino 289

Ideas 289

Incomprehensibility 320

Insight 320

Jung 309

Karl Jaspers 281, 289, 301

Life events 355

Limit situation 301

Love 345

Mayer-Gross 309

Multiperspectivalism 289
Neurosciences 330

Phenomenological philosophy 309 Phenomenology 337

Psychopathology 289, 301, 330, 337

Psychiatric practice 355

Psychotherapy 355

Relativism 289

Schizophrenia 309, 320, 330, 345

Self 320

Self-disorder 320

Social neuroscience 295

- psychology 295

Stransky 309

'Understanding' ('Verstehen') 295

Vulnerability 301 